\title{
Theoretical Studies of Quantum Interference in Electronic Transport through Carbon Nanotubes
}

\author{
W.I. BABIACZYK AND B.R. BUŁKA \\ Institute of Molecular Physics, Polish Academy of Sciences \\ Smoluchowskiego 17, 60-179 Poznań, Poland
}

\begin{abstract}
We performed studies of coherent electronic transport through a single walled carbon nanotube. In the calculations multiple scattering on the contacts and interference processes were taken into account. Conductance is a composition of contributions from different channels. We studied also spin-dependent transport in the system with ferromagnetic electrodes. The magnetoresistance is large and shows large oscillations, it can be even negative in some cases.
\end{abstract}

PACS numbers: 72.25.-b, 73.23.-b, 73.63.Fg

\section{Introduction}

Recent experiments [1-3] on electronic transport through carbon nanotubes (CNTs) show a series of interesting effects. While changing the length of the nanotube one can change a character of the transport from the classical diffusive flow to the quantum transport. The way of coupling of the CNT to the electrodes is crucial for the transport properties. If the contact resistances are large $\left(R \gg R_{Q}=13 \mathrm{k} \Omega\right)$, the transport exhibits the incoherent single-electron tunnelling character with the Coulomb blockade effect [1]. Improvement of the quality of the contacts results in an increase in the conductance of the system and one can observe the Kondo resonance, which is due to exchange interactions of conducting electrons with an uncompensated spin localized on the CNT [2]. In the case where the contacts are made very carefully and thus the coupling is strong, the transport through the system becomes coherent [3]. The interference processes and multiple scattering on the contacts are very important in this limit and lead to the Fabry-Perot interference. 


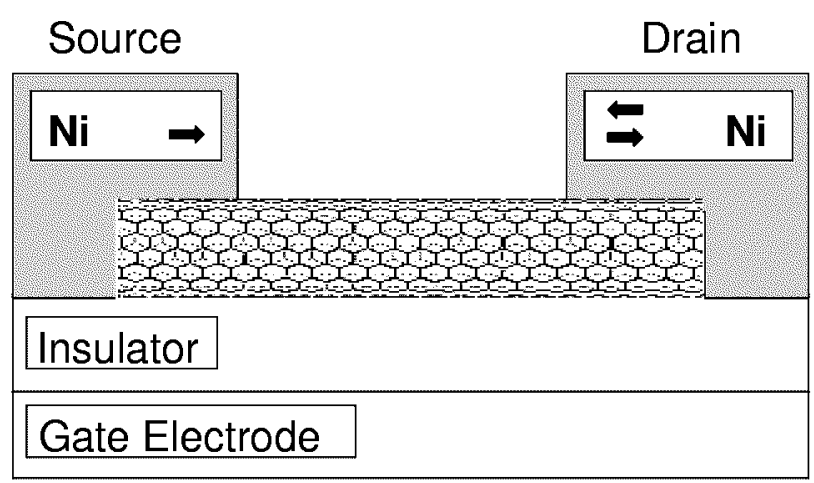

Fig. 1. A scheme of the system considered: A single walled carbon nanotube of the armchair type $(5,5)$ is attached to ferromagnetic electrodes (e.g. Ni or Co). The relative polarization in both electrodes can be changed from the parallel to the antiparallel orientation. The gate electrode voltage changes a relative energy of incident electrons transmitted through the nanotube.

We are interested in the coherent transport through molecular systems, particularly in magnetoresistance properties. Our system is presented in Fig. 1, where the single walled carbon nanotube (SWCNT) is strongly coupled to ferromagnetic electrodes (e.g. Ni). Coherent spin-dependent transport measurements were already performed on multiwalled CNT [4]. Despite the small value of the resistance $(R \geq 9 \mathrm{k} \Omega)$ it is not possible to perceive interference processes and it is very difficult to specify the mechanism of magnetoresistance. The aim of our work is to investigate the influence of the interference on the spin-dependent transport through the SWCNT in the regime of strong coupling.

\section{Description of the model and calculation of the electronic current}

We consider the SWCNTs of the armchair type, which has 5 benzene rings in the circumference. It is well known that this type of CNT has a metallic electronic structure with two conducting channels. The first and the last row of carbon atoms are connected with the electrodes, which are treated as ideal reservoirs. The system is described within the tight binding approach, in which the hopping integrals are assumed $t_{0}=-2.5 \mathrm{eV}$ for $\mathrm{C}-\mathrm{C}$ bonds. The hopping integral $t$ between the electrode and the carbon atom is treated as a parameter. The current is calculated from the time evolution of the electron number $N_{\mathrm{L}}=\sum_{i, k, \sigma} c_{k i \mathrm{~L}, \sigma}^{+} c_{k i \mathrm{~L}, \sigma}$ in the left electrode

$$
J=-e\left\langle\frac{\mathrm{d} N_{\mathrm{L}}}{\mathrm{d} t}\right\rangle=\frac{e}{\hbar} t \sum_{i, k, \sigma} \int \frac{\mathrm{d} \omega}{2 \pi}\left[G_{1 i \sigma, k i \mathrm{~L} \sigma}^{<}(\omega)+\text { c.c. }\right] .
$$

Here, $G_{1 i \sigma, k i \mathrm{~L} \sigma}^{<}(\omega)$ is the lesser Green function connecting the carbons from the first row of the nanotube with the left electrode. We sum over all incoming channels $i=1, \ldots, 10$, wave vectors $k$ and spins $\sigma= \pm 1 / 2$. The non-equilibrium Green 
functions are determined from the Dyson equation and the bare Green functions in the electrodes are taken as $g_{\alpha \sigma}=\mathrm{i} \pi \rho_{\alpha \sigma}$, where $\rho_{\alpha \sigma}$ is a density of states for electrons with the spin $\sigma$ at the Fermi energy in the left $(\alpha=\mathrm{L})$ and right $(\alpha=\mathrm{R})$ electrode, respectively. Finally, we obtain the formula

$$
J=\frac{2 e}{h} \sum_{\sigma} \frac{4}{\pi^{2} \rho_{\mathrm{L} \sigma} \rho_{\mathrm{R} \sigma}} \int \mathrm{d} \omega\left[f_{\mathrm{L}}(\omega)-f_{\mathrm{R}}(\omega)\right] \sum_{i, j}\left|G_{\mathrm{L} i, \mathrm{R} j}^{\mathrm{r}}(\omega)\right|^{2},
$$

where $G_{\mathrm{L} i, \mathrm{R} j}^{\mathrm{r}}$ is the retarded Green function connecting the channels in the electrodes and $f_{\alpha}(\omega)$ is the Fermi distribution function for electrons in the $\alpha$-electrode.

\section{Conductance for the system with paramagnetic and ferromagnetic electrodes}

Figure 2 presents the conductance $\mathcal{G}=\left.\frac{\mathrm{d} J}{\mathrm{~d} V}\right|_{V \rightarrow 0}$ calculated for the SWCNT of the length of 90 atomic layers weakly and strongly connected to the gold electrode (dashed and solid curve, respectively). For the weak coupling $\mathcal{G}$ shows sharp resonant peaks at energies $E_{n s}$ corresponding to standing electron waves in the CNT. There are two branches $(s=1,2)$ of the dispersion curves, and therefore $\mathcal{G}=\sum_{s, \sigma} \mathcal{G}_{s, \sigma}$ is a superposition of the conductances for each channel $\{s, \sigma\}$. For a resonant transmission (at $E_{n s}$ ) $\mathcal{G}_{s, \sigma}$ reaches its maximal value $e^{2} / h$. If eigenvalues $E_{n 1}$ and $E_{n 2}$ are close to each other the value of the conductance is larger than $2 e^{2} / h$ (see outer peaks on the left and the right hand side of the dashed curve in Fig. 2). Next, we increase the coupling to the value $t=1.3 \mathrm{eV}$, which gives $\mathcal{G}$ close to the experimental data $3 e^{2} / h[3]$. For this case the resonant peaks are

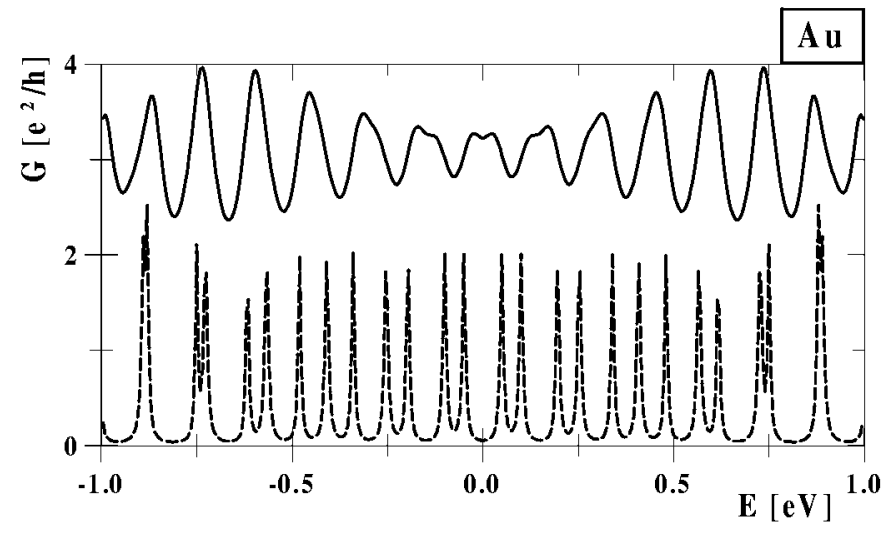

Fig. 2. Conductance $\mathcal{G}$ vs. incident electron energy $E$ for the gold electrodes calculated for $T=0$. The solid curve corresponds to the strong coupling $t=1.3 \mathrm{eV}$, and the dashed curve to the weak coupling with $t=0.4 \mathrm{eV}$. The total density of states at the Fermi energy is taken as $\rho_{\mathrm{L}}=\rho_{\mathrm{R}}=0.294$ states $/ \mathrm{eV}$ (data from [5]). 
much broader. If the peaks are close to each other, they merge together leading to a single peak with the maximal value $4 e^{2} / h$. However, the peaks can occur far from each other and then the effective conductance plot looks like at the center of Fig. 2 (close to $E=0$ ). The similar effect was observed experimentally [3]. Our approach takes into account multiple scattering on the contacts and interference processes. The electronic waves for both conducting channels corresponding to the dispersion curves $s=1,2$ are of different symmetry and a transfer matrix between them should be very small. In our opinion, interference between the channels is irrelevant. It is in contrast to [3], where a main role was assigned to the Fabry-Perot interference.

Next, we study transport through SWCNT attached to ferromagnetic electrodes. The density of states were determined by the band structure calculations performed using the tight binding version of the linear muffin-tin orbital method in the atomic sphere approximation [6]. The results are presented in Fig. 3 for the system with the Ni electrodes. The conducting channels for opposite spin orientations are different. Therefore, $\mathcal{G}$ is a superposition of four $\mathcal{G}_{s, \sigma}$ corresponding to the four different channels $\{s, \sigma\}$. Since in ferromagnetic $\mathrm{Ni} \rho_{+}=0.1897$ states $/ \mathrm{eV}$ for

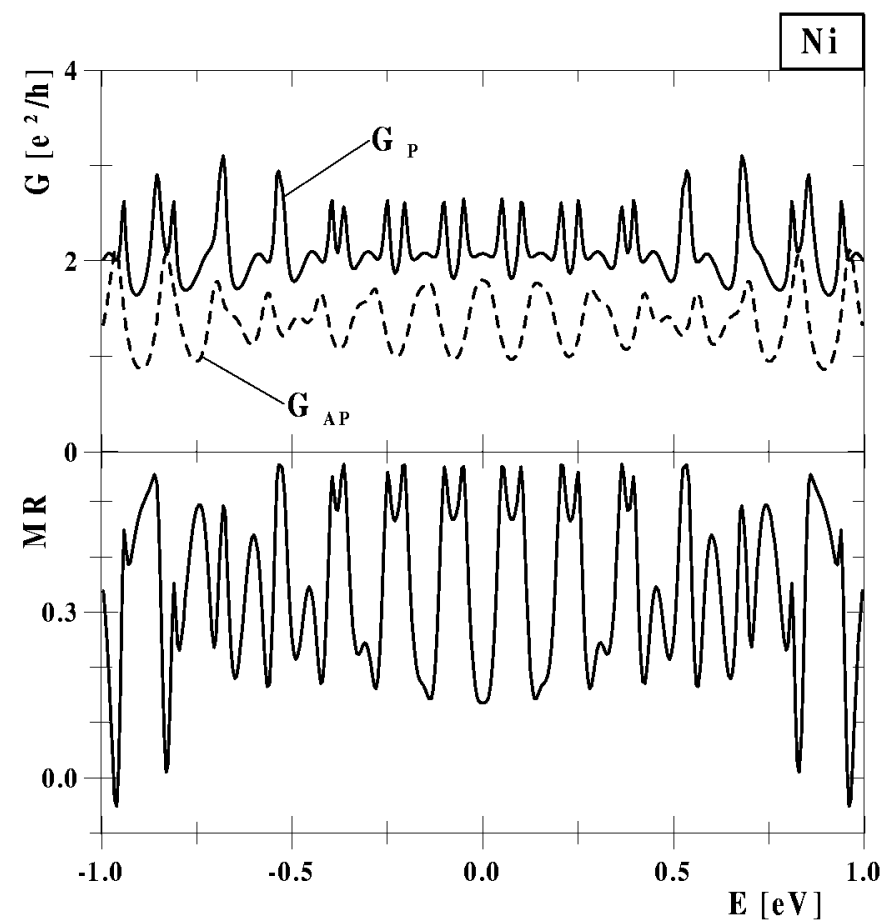

Fig. 3. The conductance (a) $\mathcal{G}_{\mathrm{P}}$ for the parallel (solid curve) and $\mathcal{G}_{\mathrm{AP}}-$ the antiparallel configuration (dashed curve) of the magnetization in the Ni electrodes, the magnetoresistance (b) plotted as a function of the energy $E$ of incident electrons. The parameters used are: $t=1.9 \mathrm{eV}, \rho_{+}=0.1897$ state $/ \mathrm{eV}$ and $\rho_{-}=1.7261$ states $/ \mathrm{eV}$. 
electrons with the up spin is much lower than $\rho_{-}=1.7261$ states $/ \mathrm{eV}$ for minority electrons with the down spin, so in the parallel configuration $(\mathrm{P})$ the conductance $\mathcal{G}_{+}=\sum_{s} \mathcal{G}_{s}$ shows broad peaks, which reach the value $2 e^{2} / h$, while $\mathcal{G}_{-}$shows sharp peaks and their maximal value is $1 e^{2} / h$. The composition of all channels results in $\mathcal{G}_{\mathrm{P}}$ with sharp peaks (solid curve in Fig. 3a). The situation is different for the antiparallel configuration (AP) $\mathcal{G}_{\mathrm{AP}}$ (see the dashed curve in Fig. 3a), where the peaks are relatively broad. The magnetoresistance $M R=\left(\mathcal{G}_{\mathrm{P}}-\mathcal{G}_{\mathrm{AP}}\right) / \mathcal{G}_{\mathrm{P}}$ is given by the relative difference of the conductance in the parallel and the antiparallel configuration. The results (presented in Fig. 3b) show large changes of the $M R$ and that the maximal value can be very large $(\max [M R]>60 \%)$.

An interesting case is the system with the cobalt electrodes. The density of states is $\rho_{+}=0.1740$ states $/ \mathrm{eV}$ for the spin $\sigma=+1 / 2$ (close to the value in $\mathrm{Ni}$ ), but $\rho_{-}=0.7349$ states $/ \mathrm{eV}$ is much smaller. The peaks of the conductance $\mathcal{G}_{-}$ are now much broader. The total conductance $\mathcal{G}_{\mathrm{P}}$ and $\mathcal{G}_{\mathrm{AP}}$ for both orientations of polarization are shown in Fig. $4 \mathrm{a} . \mathcal{G}_{\mathrm{AP}}$ is higher and shows larger oscillations

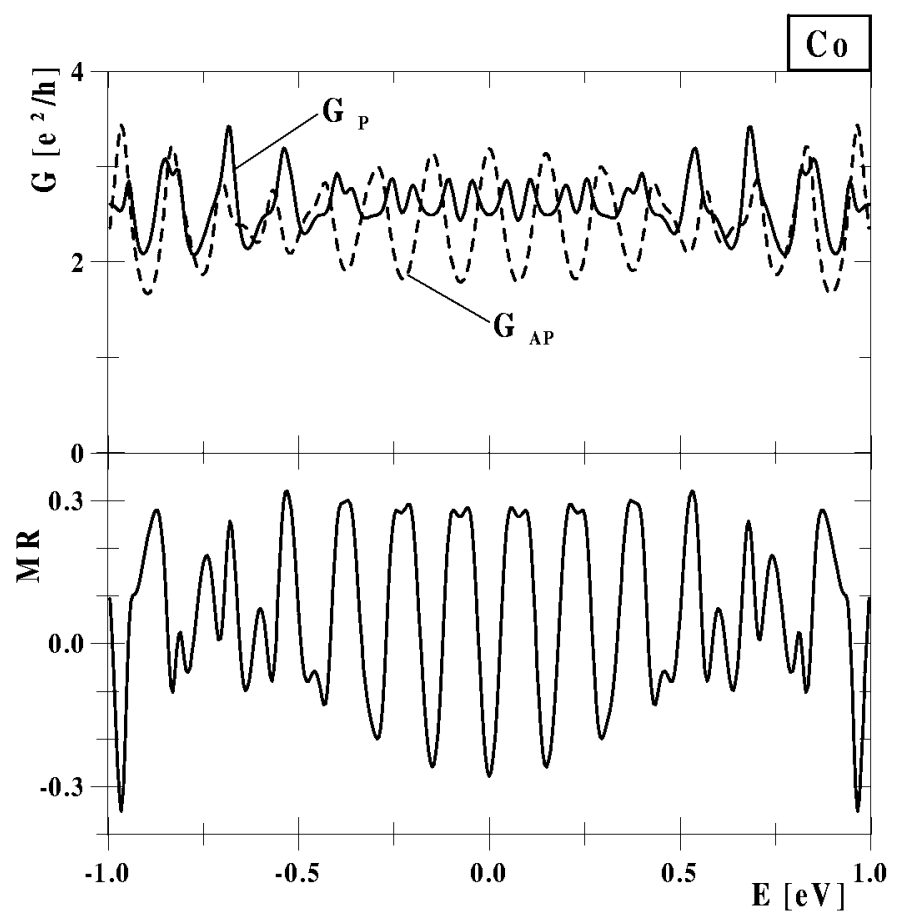

Fig. 4. The conductance (a) $\mathcal{G}_{\mathrm{P}}$ for the parallel (solid curve) and $\mathcal{G}_{\mathrm{AP}}-$ the antiparallel configuration (dashed curve) of the magnetization in the Co electrodes, the magnetoresistance (b) plotted as a function of the energy $E$ of incident electrons. The parameters used are: $t=1.9 \mathrm{eV}, \rho_{+}=0.1740$ state $/ \mathrm{eV}$ and $\rho_{-}=0.7349$ states $/ \mathrm{eV}$. 
than for Ni (compare the dashed curves in Figs. 3a and 4a). The value of the magnetoresistance (shown in Fig. 4b) is smaller, but exhibits large oscillations. It is interesting that in this case $M R$ can also be negative.

\section{Conclusions}

Our studies of coherent electronic transport through the SWCNT showed that the conductance is a composition of contributions from four channels. Although multiple scattering and interference processes were included in this approach, we could not observe any feature of destructive interference. In our opinion, the conducting channels correspond to waves of different symmetry and matrix elements between them are small. We also considered the spin-dependent transport in the system with the ferromagnetic Ni and Co electrodes. Due to a resonant nature of the electronic transport the magnetoresistance shows large oscillations and achieves large values $(\max [M R] \approx 60 \%$ and $30 \%$ for $\mathrm{Ni}$ and $\mathrm{Co}$, respectively). Since the densities of states for minority electrons (with $\sigma=-1 / 2$ ) are different in $\mathrm{Ni}$ and $\mathrm{Co}$, the magnetoresistance is different in both cases. For the Co electrodes $M R$ can even change the sign and can be either positive or negative depending on the gate voltage applied to the carbon nanotube. We believe that in near future one can produce systems with coherent contacts between ferromagnetic electrodes and the SWCNT, and that our theoretical predictions will be verified.

We would like to thank Dr. Andrzej Szajek for ab-initio calculations of the density of states for ferromagnetic metals. The work was supported by the Committee for Scientific Research (KBN) under grant No. 2 P03B 08719.

\section{References}

[1] M. Bockrath, D.H. Cobden, P.L. McEuen, N.G. Chopra, A. Zettl, A. Thess, R.E. Smalley, Science 275, 1922 (1997); S. Tans, M.H. Devoret, R.J.A. Groeneveld, C. Dekker, Nature 394, 761 (1998).

[2] J. Nygard, D.H. Cobden, P.E. Lindelof, Nature 408, 342 (2000); W.J. Liang, M. Bockrath, H. Park, Phys. Rev. Lett. 88, 126801 (2002).

[3] W.J. Liang, M. Bockrath, D. Bozovic, J.H. Hafner, M. Tinkham, H. Park, Nature 411,665 (2001).

[4] K. Tsukagoshi, B.W. Alphenhaar, H. Ago, Nature 401, 572 (1999).

[5] J.M. Seminario, C.E. De La Cruz, P.A. Derosa, J. Am. Chem. Soc. 123, 5616 (2001).

[6] A. Szajek, unpublished; G. Krier, O. Jepsen, A. Burkhardt, O.K. Andersen, The TB-LMTO-ASA Program, source code version 4.7. 